\title{
Training matters
}

\section{The case for teaching and research experience and education within basic specialist training (registrar grade) in psychiatry}

\author{
A. H. CRISP, Professor of Psychiatry, St George's Hospital Medical School, \\ London SW17 0RE
}

Medical practice can be reframed in terms of research and teaching, possessing and using the relevant knowledge, skills and attitudes. The doctor's curiosity and the diagnostic challenges of medical practice (and in particular of psychiatry) demand a capacity to think systematically, measure comprehensively and accurately and analyse the information obtained within the consultation as a preamble to management and treatment. The word 'doctor' means 'teacher' and the same communication skills are essential to both professions. Psychiatric practice is especially dependent upon them. They are also often essential for good clinical research.

The Recommendations on the Training of Specialists produced by the Education Committee of the General Medical Council (1987) identify a dozen necessary attributes of the independent medical practitioner which serve to highlight these points. The attributes include the specific capacity to advance one's subject through adding relevant knowledge and skill to it. Research skills are also basic to a number of the other attributes such as problem-solving abilities, consultation skills, self audit and the audit of others. We are now used to advising that our interventions (and those of others such as the Department of Health with its White Paper) be evaluated. It is therefore important that basic understanding of research methodology be developed in all doctors at an early stage in their specialty training. More substantial research can then be hopefully undertaken thereafter, e.g. at higher specialist training and continuing medical education levels.

Such basic understanding and ability requires education and training at registrar level, not for the selected or self selected few but for everyone.

The College provides a research option for Part II Membership examination and also recognises some time spent in the field for this purpose. However, this operates for only a small minority of candidates. The College is right to realise that its attitude to the matter is of paramount importance. There are several possible ways of developing more widespread training in research at the stage of basic specialist training:

(a) Incorporate such ideas and goals within the curriculum and examination for the Membership examination.

(b) Require that such education and training is available in all training posts/schemes. In future, research will presumably go on within all clinical practice as part of self evaluation and audit and with a greater awareness of its contribution as part of everyday clinical practice.

(c) Expect all trainees to undertake a small formal study, e.g. a case report, review of established knowledge, etc. either as a requirement of the signing up process indicating completion of training and readiness to sit the examination (e.g. by the Clinical Tutor, Regional Postgraduate Dean, Regional Professor, etc.) or as an element within part of the Membership examination itself (a major and perhaps too daunting a task for examiners, given the large numbers of candidates).

(d) Alternatively, one might substantially increase the number of research posts. These would have to be taken from the established pool of registrar posts and this would be disconcerting to the profession at large. However, it could hopefully be an occasional development.

(e) In some centres a separate research degree or taught course has been developed at Master's level. These exist in some provincial universities and sometimes overlap with MRCPsych course training in which case they may have the additional advantage of carrying required registration as part of training. There is also the MPhil (Psychiatry) in London University but this attracts only a minority of trainees and completion rates are sometimes poor. 
This statement was initially produced by me at the request of the London Professors of Psychiatry group and has since been discussed at AUTP. It is reproduced here in case it may be of interest to those organising registrar training schemes in the present climate.

\section{Reference}

Education Committee, General Medical Council (1987) Recommendations on the Training of Specialists issued in pursuance of Section 15 of the Medical Act 1978.

\title{
The role of the clinical tutor - a personal experience
}

\author{
Jefrrey R. Jones, Consultant Psychiatrist, The North Wales Hospital, Denbigh, \\ Clwyd LL16 5SS
}

The role of the clinical tutor may differ from that set out in the helpful guidelines given by the Royal College of Psychiatrists. It may differ depending on the setting of the training for instance. I was made clinical tutor some three and a half years ago for a large, mainly rural area - North Wales. The area does not have its own medical school, but is associated with the University of Wales College of Medicine, at Cardiff, some 150 miles away.

The tutor's role may, in such a setting, be expanded to include other duties apart from organising tuition, such as personnel work, counselling, organising senior registrar training or medical students' training. In addition there are certain other difficulties that arise due to the relative isolation from an academic centre. Similar problems may affect other areas of the United Kingdom and Ireland so I feel that it would be useful to describe some of my own experiences as a clinical tutor.

\section{Training scheme}

There are certain unique features in organising a training scheme for psychiatry in a large area like North Wales where hospitals at which trainees may be based are up to 60 miles apart. Additional difficulties arise when schemes involve two different health districts. When I started as clinical tutor, there was not a complete training scheme as such, although there were separate SHO and registrar posts. Following the (then) College recommendations I organised a rotational training scheme combining SHO and registrar posts with trainees appointed for 3.5 to 4 years. The scheme included training in general psychiatry based both at a large mental hospital for North Wales at Denbigh and at a new District General Hospital unit at Bangor. Mental handicap training was based at Llanfairfechan, and child psychiatry was based at the Gwynfa unit at Colwyn Bay.
I had a meeting with the new Unit General Manager, and representatives of the two districts (Gwynedd and Clwyd), when I was organising the scheme and obtained their backing and cooperation, which was necessary for such a large scheme involving rotation between the two districts. This involved such matters as accommodation and travel expenses for trainees when they moved between the two areas.

With Achieving a Balance we appear to have come full circle, as we are not now able to combine SHO and registrar training and need to separate the two.

\section{Personnel work}

One of the main problems over the last few years for the training scheme has been that of recruitment. I believe that this is a national problem which has had greater effects in our area for several reasons, including the relative unpopularity of hospital medicine, and psychiatry in particular, and the area's remoteness from the 'local' medical school. This shortage of junior staff has resulted in a number of crises and in the winter of 1986 we had six vacant posts with all the resultant disruption to services that this entails.

A number of steps were taken to deal with these difficulties. Initially two trainee posts were filled by nine session clinical assistants. Other clinical assistant sessions were provided by local GPs and other doctors and some of these were kept on for an indefinite period, although at times this has meant we have been slightly over the established number of staff. In fact a significant extra cost was incurred in paying for locum sessions, including payment for staff doing extra on-call duties. Vacant posts were continuously advertised in the British Medical Journal. Permission was obtained from the Welsh Office for some posts to attract a 'peripheral allowance' of around $£ 950$ in addition to the annual salary.

I found that much of my time was taken up in trying to recruit doctors and dealing with the conse- 